\title{
Preventive protection of marine electrical power system from the transition of generating sets to motoring mode
}

\author{
Alecsandr Saushev ${ }^{1, *}$ and Nikolai Shirokov ${ }^{1}$ \\ ${ }^{1}$ Admiral Makarov State University of Maritime and Inland Shipping, Dvinskaya Str., 5/7, Saint \\ Petersburg, 198035, Russia
}

\begin{abstract}
The approaches that ensure the trouble-free operation of marine power system in abnormal modes were considered. Such modes are usually associated with the system elements failure during operation. Particular attention was paid to the processes occurring in the circuit during transition of one of the generators to the motoring operation mode. The relevance of the considered marine power system issue was substantiated. According to the research results, using time-delay when generating a signal to disable a failed unit operating with reverse power can contribute to defect development in the primary motor. Moreover, time-delay can also lead to zero voltage in the marine electric power system. This circumstance creates the possibility of an emergency that can potentially lead to a shipping accident with the most serious consequences. The problem of timely shutdown of a faulty electrical machine before its transition to the motoring mode is defined in the research, as well as the overload prevention problem for primary motors remaining in working condition. An original diagnostic indicator was proposed based on study, which allows identifying the inoperative state of generator unit during operation. The new approach was developed, which implements the preventive control method for the marine power system in case of element failure. The forecasting of the system operation modes in case of a generator set failure, and its structural adaptation to the occurred malfunction is carried out. In contrast to the existing methods, the practical implementation of the proposed solution will allow accident-free transition of marine power system to a partially operational state without the emergency. This will have a beneficial effect on the safety of ship as a whole.
\end{abstract}

\section{Introduction}

Marine electrical power systems (MEPS) perform demanding functions for providing electrical energy to onboard power consumers. In this regard, active research is underway aimed at improving the quality indicators of electricity supplied by MEPS, as well as considering the specifics of its consumers [1-3].

\footnotetext{
*Corresponding author: saushevav@gumrf.ru
} 
Recent years have been characterized by the rapid development of various approaches implementing Smart Grid technology (intelligent grids) in order to improve efficiency of energy resources use [4, 5]. According to this concept, it is assumed expedient to place generator sets (GS) and low-voltage switchgears, also known as distributed generation plants (DGP), in close vicinity to consumers [6-8]. These plants can operate in parallel with a higher-level electric power system or independently provide power supply to the consumers assigned to them, realizing the so-called island operation mode. In this case, DGP can create energy clusters of distributed generation with parallel operation of the GS, which are similar to MEPS in the island operation mode. This feature boosts more active research in this area of the electric power industry.

In this regard, there is a great interest for projects aimed at improving the operation safety of electrical power systems in abnormal modes, related to failures of their elements during operation. One of the areas for such projects is developing methods that ensure the timely shutdown of the GS when it switches to motoring mode due to drive motor failure $[9,11]$. According to researches $[9,10]$, the formation of cutoff signal for the GS during transition to motoring mode can lead to serious primary motor damage if done with a time delay. In order to solve the issue, unique algorithms are suggested. They allow for precisely determining the reverse power occurrence moment for prompt unit shutdown. At the same time, the replacement of relays operating with a large error with modern and more accurate devices allows obtaining the required result only for the simplest electric power systems. Such systems do not include powerful consumers, which create a load comparable to the power of the generator. They also do not include hoisting machines operating with energy recovery to the grid. In most cases, short-term work in motoring mode is one of the possible options for the functioning of a working unit. In this case, implementation of suggested technical solutions will lead to the type one error during diagnosis and a possible erroneous shutdown of a serviceable GS.

According to study [12], methods of diagnosing complex systems are considered, which also consider information about the limits of their performance areas. The method of segmenting the operability area was introduced in scope of research [11] with its division into truncated areas of proper functioning, which correspond to the defined MEPS operating modes. Areas in which the GS operates as an electric motor are highlighted. For the corresponding operating modes of the electric power system the inoperable state of the GS is not identified. Such truncated areas of proper functioning were called homonymous as per the study [13]. In this case it is possible to turn off the GS that switched to the motoring operation mode without time delay. It is possible only when MEPS does not operate in the defined modes [11], or if its parameters do not belong to the homonymous area of proper functioning [13].

Evidence shows that the fundamental error of this approach lies in the excessive concentration of researchers' attention on a specific GS. The modes of MEPS functioning and changes in the parameters of other units operating in parallel are being ignored. This occurs since the main purpose of protection against reverse power is considered to be preventing serious damage to the primary drive, which is not exactly correct. In case of failure of one GS, its load is redistributed between the remaining efficient machines. At the same time, if only two units are operating initially, then the load on the operating GS more than doubles, taking into account the reverse power. As a rule, this leads to stopping the primary drive due to the overload and to an interruption in the power supply to consumers. Such interruption can cause disruptions in technological processes and large economic losses, for example, during the extraction of minerals.

Equally serious problems can occur in the navy. Thus, for example, the recovery time for the power supply of the ship's electrical networks after a power outage ranges from several tens of seconds to several minutes [14]. During this time the ship is deprived of 
control, and often of propulsion. If a similar situation arises during a storm or in a bottleneck, while maneuvering in the reef area or near coastal rocks, there is a real danger of a shipwreck and death of crew members.

Above-mentioned circumstances confirm the importance and relevance of studies aimed at timely identification of the inoperative GS state, network unload and shutdown of failed machine before it switches to the motoring mode.

\section{Materials and Methods}

In order to solve this problem, the preventive control (PC) method was used. According to the research [11], it is the process of forming the impact on MEPS. As a result of such impact, technical state of protection will belong to the truncated area of correct functioning after protection is triggered. The structural or parametric adaptation of the system to the arisen malfunction occurs during this process, which is followed by accident-free transition to a partially operable state. In this regard, the issue of determining the technical state of MEPS becomes relevant and comes down to determining its operability or inoperability. The PC method is applicable in case of system's transition to inoperative state. In order to assess the state of MEPS, it is convenient to use the concept of operability area $(G)$. It is understood as the set of permissible values of the primary parameters $\mathbf{X}$, for which all the requirements for system output parameters $\mathbf{Y}$ and output parameters of its functional blocks $\mathbf{Z}$ are fulfilled [12]. The set $G$ can be represented as the intersection of sets $D_{x}, M_{z}$ and $M_{y}$ :

$$
G=D_{x} \cap M_{z} \cap M_{y}
$$

where $D_{x}$ is the tolerance box of the primary parameters, which has the bar shape and in Euclidean space can be described as $D_{x}=\bigcap_{k=1}^{n} D_{k}, D_{k}=D_{k \min } \cap D_{k \max }$. The tolerance box corresponds to the internal operability condition. $M_{z}$ is the area generated by the space display of the primary parameters $\Phi_{z x}: D_{z} \rightarrow M_{z}, M_{z}=\bigcap_{r=1}^{p} M_{r}$ of the tolerance box of the system's functional block parameters $D_{z}=\bigcap_{r=1}^{p} D_{r}$. This tolerance box corresponds to the internal operability condition. $M_{y}$ is the area generated by the space display of the primary parameters $\Phi_{y x}: D_{y} \rightarrow M_{y}, M_{y}=\bigcap_{i=1}^{m} M_{i}$ of the output parameter tolerance area of the MEPS, which is corresponding to the external operability condition [12].

Along the lines with the study [15], GSs operating in parallel as an autonomous generating system (AGS) were considered. The system's elements are GSs themselves, interconnected and intended to supply the ship with electrical energy of the required quality. Since the analysis depth in current task is determined by the parameters of the GS, which are main elements of the system, and there are no larger functional blocks, then in this case the below is true:

$$
G=D_{x} \cap M_{y}
$$


In order to determine the AGS operability, it is necessary and sufficient that the point in the space of primary parameters $S(\mathbf{X})$, which characterizes its technical state, belongs to the area $G$. Then the condition for the AGS operability is written as follows:

$$
S(\mathbf{X}) \in G
$$

The AGS operability area defines the set of permissible values of its internal (primary) parameters, at which all the requirements for the system's output parameters are fulfilled [12-16]. On the other hand, according to regulatory documents the object's state when it is capable of performing the required functions is called workable. In other words, the GS can be workable, but disabled by the protection. Therefore, the operable state concept should be used only at the first stage of PC for diagnosing AGS. In case of failure of at least one power source, it is necessary to determine the mode system will function in after the protection is triggered and the inoperative GS is disabled. In addition, it is necessary to form a control action aimed at a trouble-free transition of the MEPS to a partially operable state. Thus, it is necessary to transfer the system to the operation mode, in which it will perform the required functions without the emergency situations associated with the ship's power outage, loss of its controllability and progress. In this regard, it is advisable to throw in the area $H$ that characterizes the presence of the AGS in both operable and working condition. Then, for the system to be efficient and to perform the required functions, it is necessary and enough that the point $S(\mathbf{X})$ characterizing its technical state belongs to the area.

The area $H$ is the set of admissible values of the internal and input parameters of the MEPS, at which all the requirements for the system's output parameters are fulfilled. In other words, the performance area $H$ in the narrow sense is the intersection of areas $D_{x}$, $M_{y}, M_{u}$ :

$$
H=D_{x} \cap M_{y} \cap M_{u}
$$

where $M_{u}$ is the area generated by the mapping of the primary parameters $\Phi_{u x}: D_{u} \rightarrow M_{u}, M_{u}=\bigcap_{c=1}^{e} M_{c}$ into the space of tolerance box $D_{u}=\bigcap_{c=1}^{e} D_{c}$ of control actions area [11].

Truncated areas of proper functioning $\left(w_{j}^{q}\right)$ are the areas obtained after segmentation of the performance area $(H)$ in the narrow sense. The process of dividing the $H$ area into separate segments with homogeneous features, which for example correspond to the state of the proper MEPS functioning, is called the segmentation of the performance area of in [11]. The following formulae are obtained in this case:

$$
\forall w_{j}^{q} \in H \text { and } H=\bigcup_{j=1}^{q} w_{j}^{q}, j=\overline{1, q} .
$$

If at least one of the parallel operating GSs fails, requirements (1-3) will not be met. However, for a trouble-free transition to a partially operable state, it is necessary that the point $S(\mathbf{X})$ (characterizing the AGS state) belongs to one of the truncated regions of proper functioning $w_{j}^{q}$ as a result of PC. The condition for the trouble-free operation of the MEPS in case of element failure can be written as follows: 


$$
S(\bar{X}) \in w_{j}^{q}=D_{x}^{q} \cap M_{y}^{q} \cap M_{u}^{q}, \quad j=\overline{1, q},
$$

where $D_{x}^{q} \in D_{x} ; M_{y}^{q} \in M_{y} ; M_{u}^{q} \in M_{u}$.

Operable GSs in the considered mode will be operable in all AGS operation modes, including the mode enabled after the inoperative unit is turned off. Thus, in order to fulfill condition (4) it is necessary to check whether the set of input (control) signals $D_{u}=\bigcap_{c=1}^{e} D_{c}$ meets the specified requirements. In this case $e=1$, the control signal is the network active load $P$. In this case, the following inequality must be satisfied:

$$
P \leq P_{\lim j}=k \bigcup_{i=1}^{n} N_{i}
$$

where $P_{\lim j}$ is the permissible active load value in the $j$ - th AGS operating mode. $N_{i}$ is the highest power that can be developed by the $i$ - th GS (the rated power, for example). $n$ is the number of working efficient GS; $k$ is a coefficient taking into account the uneven loading of the GA.

Based on the suggested approach, in case of gradual MEPS failure and identifying the inoperative state of at least one GS operating in parallel, it is necessary to assess the power AGS can generate after disabling the failed units and compare it with the real load. If a decrease in the generated power leads to an operable GS overload, then it becomes necessary to turn off the groups of consumers until the moment when faulty machines are turned off. In this case, after the protection devices are triggered, the remaining operational units will not be overloaded. The MEPS will switch to a partially operational state without interruption in the ship's power supply. The duration of the AGS diagnostics $\left(t_{1}\right)$ in this case is a particularly relevant indicator of the PC quality. According to the definitions provided in the regulatory documents, AGS diagnostics duration is the time interval required to identify the inoperative state of the operating GS. This indicator will be further called the operational duration of the defect search, that is, the time interval from the malfunction occurrence till its detection.

\section{Results}

Let us consider the method of protecting the GS from the transition to the motoring mode, which is widely used by companies across the world. According to this method, the reverse power value is used as a diagnostic indicator $[9,10]$. One of the operating GSs might fail, for example, in case of a failure of the fuel supply system to the diesel engine. In such case the unit will continue to operate, generating fuel residues in pipelines and filters. In such situation the machine will be unloaded, and its load will be redistributed between the remaining operational GSs.

The idea of implemented approach is that the moment when the amount of power consumed by the generator exceeds the specified value and the GA is disconnected from the network with a time delay. At the same time, in accordance with the actual norms for diesel generators, the reverse power value can reach $15 \%$ of the nominal value. The time delay when triggered is 10 seconds (for carrier ships it is usually 5-6 seconds) [15].

The diagnostic duration $t_{1}$ can be determined as follows: 


$$
t_{1}=t_{2}+t_{3}+t_{4}
$$

where $t_{2}$ is the time interval from the defect occurrence till the complete unloading of the failed GA. $t_{3}$ is the time interval from complete unloading of the inoperative GA until the moment the reverse power exceeds the permissible value. $t_{4}$ is the interval corresponding to the time delay when triggered.

In this case, the $t_{2}$ value depends significantly on the nature and location of the defect, the parameters of the primary driver, the magnitude of the MEPS load and usually varies in the range from 1 to 20 seconds. The $t_{3}$ time interval is usually 1-2 seconds; the $t_{4}$ duration is usually $5-6$, but not more than 10 seconds.

Thus, the diagnostic duration for protecting the GS from reverse power usually ranges from 8-9 to 30 seconds. The response time is several orders less for the equipment, so this value can be neglected. Before the protection is triggered, the remaining operational units are additionally loaded with the load of the failed GS and reverse power, which can lead to their overload and network disconnection.

In case methods of preventive protection of MEPS against reverse power are implemented $[11,13]$, the diagnostics duration will be equal to the unloading time of the inoperative unit, that is $t_{1}=t_{2}$.

It is possible to avoid additional network loading with reverse power by following this approach, but the possibility of overloading operable GA as a result of load redistribution remains. Therefore, devices implementing the approaches considered in studies $[11,13]$ are advisable to use in fairly simple electric power systems with a relatively low adaptation degree to potential defects [15].

Based on the above-mentioned, the effective preventive MEPS protection from the operation of GS in the motoring mode is possible. It is necessary to identify the inoperable source of electrical energy earlier than the overload of the operable GS occurs. The following condition has to be met:

$$
t_{1} \leq t_{2.1}
$$

where $t_{2.1}$ is the time interval between defect occurrence and the overload moment of any of the operable GSs, reduced by the time of MEPS adaptation to the malfunction. In this case $t_{2.1} \in t_{2}$.

The parameter $t_{2.1}$, as well as the parameter $t_{2}$, depends on the location and nature of the defect, on the primary drive parameters, as well as the network load and can vary widely. Therefore, the PC diagnostic indicator has to take into account these correlations. In this regard, at the moment when the unevenness of the GS load goes beyond the setting limits, the generator set with decreased load is determined as inoperative. At the same time the load of another generator increases.

The inoperative state of the GS with decreased load was denoted as $F_{i}, i=1,2 \ldots n$, where $n$ is the number of generators currently operating. Then the diagnostic indicator for determining the GS inoperative state for PC purposes can be presented in the following form:

$$
F_{i}=L_{1} \wedge\left(L_{2}>L_{\text {lim }}\right)
$$


where $L_{1}$ is the condition for reducing the load of one of the working GSs when the load of the other GSs is increased. The inequation $L_{2}>L_{\text {lim }}$ is true when the difference between the active loads of the generators is greater than the permissible value.

Expression (6) allows relatively simply determining the inoperative state of the GS as part of the AGS, until the remaining units operating in parallel are overloaded. In this case, condition (5) is fulfilled, which means that the problem of technical diagnostics for PC purposes is resolved. At the same time, the actual use of expression (6) as a diagnostic indicator in some cases can lead to the first kind error and shutdown of an operable unit. According to the study [15], GSs can operate in manual mode with different power, in such case the condition $L_{2}>L_{\lim }$ will be met. When the AGS switches to automatic operation, the loading of the units will be leveled, so the load for one of them will decrease, and will increase for the other. This will lead to erroneous identification of the unloaded GS as inoperative one and it will be disconnected from the network. The truncated area of proper functioning, which corresponds to this MEPS operation mode, is designated as $w_{5}^{q}$ [15]. A similar situation occurs when one of the generators is switched on in parallel. After closing the automatic switch of the synchronized GS and pulling the generator into synchronism, the connected machine is loaded while the generators operating in the network are simultaneously unloading. In this case, condition (6) is fulfilled and all previously operating units will be recognized as inoperative and turned off. The truncated area of proper functioning, which corresponds to the mode of switching on the GS for parallel operation, is designated as $w_{1}^{q}$ [15]. Consequently, condition (6) can be successfully applied for PC purposes only when the generators are already operating in parallel in automatic mode.

The truncated area of correct functioning $w_{1}^{q}$ and $w_{5}^{q}$, characterized by a diagnostic indicator, for example, condition (6), were called homonymous areas [13]. If the point $S(\mathbf{X})$ belongs to such area, then the control devices will record an inoperative state of an operable object. Therefore, the operation modes corresponding to these areas should be excluded from the diagnostic process. In this regard, it was suggested [15] to identify the MEPS inoperative state if the following logical conditions are met:

$$
F_{i}=L_{1} \wedge\left(L_{2}>L_{\lim }\right) \wedge\left(\overline{w_{1}^{q}} \vee \overline{w_{5}^{q}}\right)
$$

Thus, according to the research [5], if the load for one of the GSs starts to decrease during the MEPS operation, the unit to be unloaded is identified as inoperative. In such case the load for other GSs will grow, and the difference in loads exceeds the permissible value and at this moment there is no synchronization or switching from manual to automatic MEPS operation mode. Implementing the diagnostic indicator (7) considered in the research [15] makes it possible to identify the inoperative state of a failed machine in a timely manner, before the overload of working GSs. In this case, condition (5) is fulfilled and this method can be applied to the PC. At the same time, a relative disadvantage of this approach is the need to use specific mode sensors, which correspond to $w_{1}^{q}, w_{5}^{q}$. Moreover, the communication lines should be used, which can cause certain difficulties in practical implementation and slightly reduces the reliability of diagnostic tools.

By making some changes to the diagnostic indicator (6), the situation can be significantly improved. To do this, it is enough to exclude the identification process for homonymous areas by replacing them with a general diagnostic parameter. In this regard, the attention is drawn to the fact that when GSs loads change multidirectional, its difference 
decreases in the modes corresponding to $w_{1}^{q}$ and $w_{5}^{q}$. The parameter $L_{3}$, which corresponds to the process of increasing the load difference in of the aggregates, was introduced. At the same time, the sensors are not used for determining the processes of switching on the GSs for parallel operation, as well as for switching the MEPS from manual mode to automatic. The diagnostic sign of a failed GS can be written as a following logical expression:

$$
F_{i}=L_{1} \wedge\left(L_{2}>L_{\text {lim }}\right) \wedge L_{3}
$$

Thus, if load of one of the GSs begins to decrease during the operation of the MEPS, while for other (others) it grows, then the unloaded unit will be identified as inoperative. This is true for situations when the difference in loads increases and exceeds the permissible value. For the practical implementation of diagnostics, considering the feature characterized by expression (8), it is possible to use only standard information coming from the GS load sensors. This allows significantly simplifying the determination of the technical state of operating units and to increase the operation reliability of technical diagnostics for PC purposes.

\section{Discussion}

As a rule, one of the GSs switches to the motoring mode as a result of MEPS malfunction, but sometimes the number of failed units can be greater, in general it is equal $m(m=1,2 \ldots n-1)$. Having determined the number of failed power sources on the basis of (8), it is possible to predict the MEPS operating mode after the protection is triggered and identify the need to unload the network [11]. The condition for disconnecting consumers can be formulated as follows:

$$
N_{m-n} \leq P
$$

where $N_{m-n}$ is the generated power value of GSs, which still function after the protection of working GS is activated.

Thus, the network unload is carried out before the disconnection of the inoperative units, and only if the total MEPS load exceeds the calculated value of the permissible AGS load in case of disconnected inoperative GSs. If the power reserve is large enough and condition (9) is not met, then you can start disconnecting and stopping the inoperative machines. Otherwise, it is advisable to calculate the missing capacity of the AGS in the new MEPS operating mode and turn off the group of consumers, for example, considering their weight factors. Then the network load will decrease, and the system's technical condition will belong to the area of correct functioning $w_{n-m}^{n}$ after disconnecting the failed units. In this case condition (4) will be fulfilled, overloading of operational GSs will not occur, the transition of the MEPS to a partially operational state will be carried out without an emergency.

\section{Conclusion}

Modern systems for protecting MEPS from the GS transition into the motoring mode, which use the reverse power value for a given time as a diagnostic feature, in most cases turn out to be ineffective. Their use can lead to network overload, de-energizing the ship, losing its controllability and propulsion. 
For an emergency-free transition to a partially operational MEPS state in case of failure of at least one of the power sources, it is advisable to use the preventive control method. In this case, it is necessary that as a result of the PC, the point in the space of primary parameters $S(\mathbf{X})$, which characterizes the state of the system, belongs to one of the truncated areas of proper functioning $w_{j}^{q}$. That is, condition (4) has to be satisfied.

In order to control the MEPS efficiently in abnormal modes associated with GS failure, in accordance with the condition (6) it is necessary that identifying the inoperative source of electrical energy is carried out earlier than the overload of the operable GSs occurs.

The suggested method of preventive protection against the transition of GS into the motoring mode allows for an emergency-free transition of the system to a partially operable state. It increases the reliability and survivability of the MEPS when operating in emergency situations.

\section{References}

1. Seong-Hoon Kim, Myung-Il Roh, Min-Jae Oh, Sung-Woo Park, In-Il Kim, International Journal of Naval Architecture and Ocean Engineering 12, 440-454 (2020) DOI: 10.1016/j.ijnaoe.2020.03.007

2. A.V. Saushev, S.E. Kuznetsov, A.B. Karakayev, IOP Conf. Series: Earth and Environmental Science 194(8) (2018) DOI: 10.1088/1755-1315/194/8/082037

3. Niki Sadat Abbasian, Afshin Salajegheh, Henrique Gaspar, Per Olaf Brett, Journal of Industrial Information Integration 10, 29-38 (2018) DOI: 10.1016/j.jii.2018.02.002

4. G. Dileep, Renewable Energy 146, 2589-2625 (2020) DOI: 10.1016/j.renene.2019.08.092

5. Shuva Paul, Md Sajed Rabbani, Ripon Kumar Kundu, Sikdar Mohammad Raihan Zaman, 1st International Conference on Non Conventional Energy (ICONCE 2014) IEEE, 200-203 (2014)

6. Al Rammal Zeina, Nivine Abou Daher, Hadi Kanaan, Imad Mougharbel, Maarouf Saad, 4th International Conference on Renewable Energies for Developing Countries (REDEC) IEEE, 1-5 (2018) DOI: 10.1109/REDEC.2018.8597975

7. Rupa Mishra, Udayan Banerjee, Thotakura N.S.C. Sekhar, Tapas Kumar Saha, IET Electric Power Applications 13.10, 1497-1506 (2019) DOI: 10.1049/iet-epa.2018.5882

8. Y.N. Bulatov, Proceedings of Irkutsk State Technical University 21.10(129), 78-94 (2017) DOI: 10.21285/1814-3520-2017-10-78-94

9. H. Yaghobi, IET Electric Power Applications 12.4, 508-517 (2018) DOI: 10.1049/ietepa.2017.0491

10. Mehdi Samami, Milad Niaz Azari, IET Electric Power Applications 13.12, 2128-2138 (2019) DOI: 10.1049/iet-epa.2018.5961

11. N.V. Shirokov, Vestnik Gosudarstvennogo universiteta morskogo i rechnogo flota imeni admirala S.O. Makarova 11.2, 396-405 (2019) DOI: 10.21821/2309-5180-201911-2-396-405

12. A.V. Saushev, N.V. Shirokov, Vestnik Gosudarstvennogo universiteta morskogo i rechnogo flota imeni admirala S.O. Makarova 2(36), 143-156 (2016) DOI: 10.21821/2309-5180-2016-8-2-143-156

13. N.V. Shirokov, Vestnik Gosudarstvennogo universiteta morskogo i rechnogo flota imeni admirala S.O. Makarova 12.2, 390-401 (2020) DOI: 10.21821/2309-5180-202012-2-390-401 
14. N.N. Portnyagin, S.U. Trudnev, Vestnik Kamchatskogo Gosudarstvennogo Tekhnicheskogo Universiteta 18, 32-35 (2011)

15. A. Saushev, N. Shirokov, S. Kuznetsov, Advances in Intelligent Systems and Computing 1258 AISC, 388-398 (2021) DOI: 10.1007/978-3-030-57450-5_33

16. D.A. Nazarov, Informatika i sistemy upravleniya 4(54), 94-104 (2017) DOI: 10.22250/isu.2017.54.94-104 